\title{
Implementation of Low Voltage Sub-Bandgap Voltage Reference in 90nm CMOS
}

\author{
Ritesh Soni \\ M.Tech in ECE \\ Department of Electronics and Communication \\ Engineering \\ Goverment Engineering College, \\ Idukki Kerala, India
}

\author{
Prof. Sumam M J \\ Associate Professor \\ Department of Electronics and Communication \\ Engineering \\ Goverment Engineering College, \\ Idukki Kerala, India
}

\begin{abstract}
The paper introduces a new low voltage design Sub-bandgap reference circuit consists of the MOSFETs and BJT subthreshold. The proposed sub-BGR circuit shall be implemented in a standard CMOS technology of $90 \mathrm{~nm}$. The results achieved low power consumption in sub-BGR circuit and operated from $0.7 \mathrm{v}$ to $2 \mathrm{v}$ supply voltage. The simulation was carried out at voltage variations and temperature variation at range of $-20^{\circ} \mathrm{C}-140^{\circ} \mathrm{C}$ on the cadence virtuoso using spectre simulator.
\end{abstract}

Keywords-Subthreshold, voltage reference, Bandgap reference, Temperature coeffificient

\section{INTRODUCTION}

The increase in portable device usage has boosted to emphasis on minimizing power, speed and area. With the advancement of the semiconductor manufacturing technology the feature size of the devices had been reduced to nanometers. More devices are integrated on the same area of the chip, thus significantly improving system performance. The CMOS subthreshold voltage references (VRs) were operated with low supply voltage and consumed low power [1]. The VRs can be categorized as two types: one type relating to the bandgap voltage of Silicon or a fraction of it (i.e., subbandgap) with BJTs and resistors, The other was related to other factors such as MOS transistor threshold voltages and MOS transistor subthreshold parameters (For resistors with or without [1]-[4]. Although several low-power VRs were developed in [1]-[20], the other output voltage either increases exponentially or decreases linearly with temperature. The most popular onchip integration solution is the Bandgap Voltage Reference (BGR), which can be used in standard CMOS technology that exploits parasitic vertical BJTs. Conventional BGRs produce an independent reference for low temperature, approximately $1.25 \mathrm{~V}$ and thus require a higher supply voltage that may not satisfy the low-voltage requirements for low-power applications. However, many approaches have been introduced using the BGR concept to maintain sub-1V Operation [3]. Bandgap references (BGRs) are widely used for generating a temperature-insensitive reference voltage determined by the bandgap of silicon[5]-[6]. The BGR typically uses PN diodes to produce both proportional-toabsolute (PTAT) and complementary-to-absolute (CTAT) temperature. The circuits given in [6] and [7] The switched- capacitor voltage divider and charge pump were used to achieve a complementary-to absolute-temperature division (CTAT) voltage (VBE) and low voltage power supply, but the temperature coefficient (TC) is as high as $75 \mathrm{ppm} /{ }^{\circ} \mathrm{C}$ and the temperature range is limited to be only $0^{\circ} \mathrm{C}-80^{\circ} \mathrm{C}$ [7]. CMOS VRs [8]-[12] has several issues remaining, such as leakage current consumption, low reference voltage of $257.5 \mathrm{mv}$ and wide area. In this work, we propose a CMOS subthreshold voltage reference (VR) implemented in a circuit. The reference voltage is biased with the temperature-independent, increasing performance and accuracy of circuit with very low sensitivity to variations in process, supply voltage. For the rest of the paper, Section II present the materials and methods of the voltage reference. The measurement results of a prototype design in a standard $0.90-\mu \mathrm{m}$ CMOS process are introduced and analyzed in Section III.

\section{PROPOSED VOLTAGE REFERENCE}

Fig. 1 shows the proposed sub-BGR circuit using a single parasitic vertical PNP BJT, i.e., $\mathrm{Q}_{0}$ to generate CTAT Voltage and poly-resistance $R_{2}$ for PTAT voltage generation. The circuit process can be described as the summation of two currents (one PTAT and the other CTAT). BJT 's base emitter forward voltage indicates a negative TC, for a bipolar system collector current $\mathrm{I}_{\mathrm{C}}$ and saturation current $\mathrm{I}_{\mathrm{S}}$ depends on temperature as shown below

$$
\begin{aligned}
& I_{C}=I_{\mathrm{s}} \exp \left(\frac{\mathrm{V}_{\mathrm{BE}}}{\mathrm{V}_{\mathrm{T}}}\right) \\
& \mathrm{I}_{\mathrm{S}}=\mathrm{bT}^{(4+\mathrm{m})} \exp \left(\frac{\mathrm{E}_{\mathrm{g}}}{\mathrm{KT}}\right)
\end{aligned}
$$

The current $I_{R}$ flowing through the resistor $R_{2}$ is given by

$$
I_{R}=\frac{V_{B E}}{R_{2}}
$$

where $\mathrm{VBE}$ is the emitter-base voltage of $\mathrm{Q}_{0}$ as shown in equation (3), the voltage $V_{B E}$ should be decreased and $R_{2}$ increased in order to reduce the current $I_{R}$ and thus the power consumption. Although, improving $\mathrm{R}_{2}$ will eventually increase the chip area consumption. So the best way to do that is to 
minimize the $\mathrm{V}_{\mathrm{BE}}$ value. Decreasing the $\mathrm{Q}_{0}$ current often reduces its base-emitter voltage. Reducing $\mathrm{V}_{\mathrm{BE}}$, however, also where $\mathrm{V}_{\mathrm{BE}}$ is the emitter-base voltage of $\mathrm{Q}_{0}$ as shown in equation (3), the voltage $V_{B E}$ should be decreased and $R_{2}$ increased in order to reduce the current $I_{R}$ and thus the power consumption. Although, improving $\mathrm{R}_{2}$ will eventually increase the chip area consumption. So the best way to do that is to minimize the $\mathrm{V}_{\mathrm{BE}}$ value. Decreasing the $\mathrm{Q}_{0}$ current often reduces its base-emitter voltage. Reducing $\mathrm{V}_{\mathrm{BE}}$, however, also reduces the negative $\mathrm{TC}$ or increases the absolute value of $\mathrm{V}_{\mathrm{BE}} \mathrm{TC}$ since $\mathrm{V}_{\mathrm{BE}}$ derivative analysis is given as

$$
\begin{aligned}
& \frac{\delta \mathrm{V}_{\mathrm{BE}}}{\delta \mathrm{T}}=\frac{\mathrm{V}_{\mathrm{T}}}{\mathrm{T}} \ln \frac{I_{C}}{I_{S}}-(4+\mathrm{m}) \frac{\mathrm{V}_{\mathrm{T}}}{\mathrm{T}}-\frac{\mathrm{E}_{\mathrm{g}}}{\mathrm{KT}^{2}} \mathrm{~V}_{\mathrm{T}} \\
& \frac{\delta V_{B E}}{\delta T}=\frac{V_{B E}-(4+m) V_{T}-E_{g} / q}{T}
\end{aligned}
$$

where $\mathrm{m}$ is the nonidentical factor of transistor, $\mathrm{V}_{\mathrm{T}}$ is the thermal voltage, Eg is the bandgap energy of silicon, and $\mathrm{q}$ the elementary charge. The current through $\mathrm{Q}_{0}$ should not be too small so as not to increase the required positive TC of the proportional-to-absolute-temperature (PTAT) voltage. To optimize the power and area, the current $I_{R}$ through $R_{2}$ is chosen to be three times of the current through $\mathrm{Q}_{0}$. The combination of the PMOS with a $\mathrm{P}+$ poly resistor $\mathrm{R}_{2}$ produces a PTAT current flow. Reference voltage with nominally zero temperature coefficient is obtained with negative and positive TC voltage. The circuit is designed using 90nm CMOS technology.

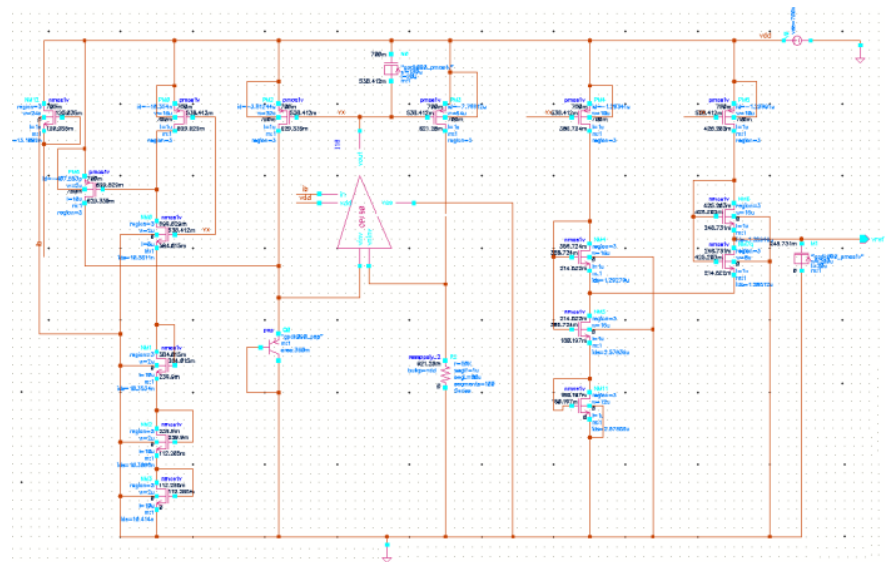

Fig. 1. Schematic of the proposed sub-BGR

The operational amplifier OPA shown in Fig. 2 enforces that nodes to have equal potential. The OPA uses the folded cascode structure to improve the loop gain and Power. The offset voltage of the OPA can be minimized by using large-size input transistors and coupling capacitor. The transistors $\mathrm{PM}_{2}-\mathrm{PM}_{3}$ are current mirrors and the size of $\mathrm{PM}_{3}$ is approximately two times of $\mathrm{PM}_{2}$ similarly $\mathrm{PM}_{4}-\mathrm{PM}_{5}$. The transistor $\mathrm{NM}_{11}$ work as diode connected NMOS, So the current through $\mathrm{NM}_{11}$ is the same as that of $\mathrm{R}_{2}$.

The PTAT voltage generator consists of transistors $\mathrm{NM}_{4}$ $\mathrm{NM}_{7}$ all of which work in the subthreshold region. The subthreshold drain current $I_{D}$ of a MOSFET is an exponential function of its gate source voltage $V_{G S}$ and drain source voltage $\mathrm{V}_{\mathrm{DS}}$.

For $V_{D S}>0.1 \mathrm{~V}$, drain current $I_{D}$ is almost independent of $\mathrm{V}_{\mathrm{DS}}$ and is approximately given by

$$
\begin{gathered}
\mathrm{I}_{\mathrm{D}}=\mathrm{K} \mathrm{C}_{\mathrm{ox}}(\eta-1) \mathrm{V}_{\mathrm{T}}^{2} \exp \left(\frac{V_{G S}-V_{T H}}{\eta V_{T}}\right) \\
\Delta \mathrm{V}_{\mathrm{GS}}=\mathrm{V}_{\mathrm{GS} 5}-\mathrm{V}_{\mathrm{GS} 4}=\eta \mathrm{V}_{\mathrm{T}} \ln \left(\frac{\mathrm{nK}_{4}}{\mathrm{~K}_{5}}\right)
\end{gathered}
$$

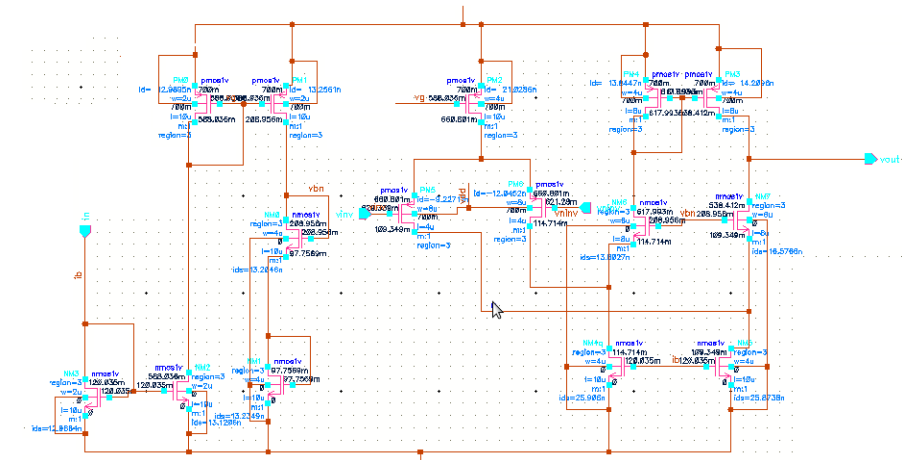

Fig. 2. Schematic of the OPA used in the Sub-BGR circuit.

where $\mathrm{K}$ is the aspect ratio $(\mathrm{W} / \mathrm{L})$ of the transistor, $\mu$ is the carrier mobility, $\mathrm{C}_{\mathrm{OX}}$ is the gate-oxide capacitance, $\mathrm{V}_{\mathrm{T}}$ is the thermal voltage, $\mathrm{V}_{\mathrm{TH}}$ is the threshold voltage of the MOSFET, and $\eta$ is the subthreshold slope factor. The drain source voltage $\mathrm{V}_{\mathrm{DS}}$ of transistors $\mathrm{NM}_{4}-\mathrm{NM}_{7}$ are designed to be larger than $0.1 \mathrm{~V}$. The threshold voltages of $\mathrm{NM}_{4}$ and $\mathrm{NM}_{5}$ are the same, and the drain current of $\mathrm{NM}_{5}$ is two times that of $\mathrm{NM}_{4}$, so the drain-source voltage of $\mathrm{NM}_{5}$ can be derived from (6) is given by

$$
V_{D S 5}=\Delta V_{G S}=V_{G S 5}-V_{G S 4}=\eta V_{T} \ln \left(\frac{n K_{4}}{K_{5}}\right)
$$

In a similar way, the voltage $V_{D S 7}$ is given by

$$
V_{D S 7}=\Delta V_{G S}=V_{G S 7}-V_{G S 6}=\eta V_{T} \ln \left(\frac{n K_{6}}{K_{7}}\right)
$$

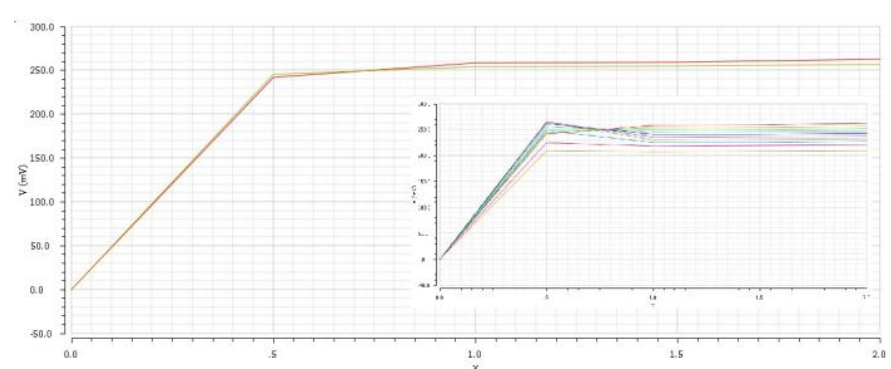

Fig. 3. Practically measured $V_{\text {ref }}$ with respect to change in supply voltage. 
Fig 3. Shows the simulated output voltage determined against $0 v$ to $2 v$. Fig. 3. Simulated output voltage measured was also shown by temperature variation $-20^{\circ} \mathrm{C}-140^{\circ} \mathrm{C}$. The generated $\mathrm{V}_{\text {ref }}$ of the proposed sub-BGR is $248 \mathrm{mv}$ and also associated in the PTAT term with the process parameter $\eta$ in the PTAT term ,which is not constant in actual system and depends on the capacitance of the gate-oxide and depletion layer. Still the variance is not too large. The TC of $\mathrm{V}_{\text {ref }}$ is also related to the process parameter and the effect of parameter $\eta$ on $V_{\text {ref }}$ and $\mathrm{TC}$ of $\mathrm{V}_{\text {ref }}$ are similar, so output $\mathrm{V}_{\text {ref }}$ variation is very small . Therefore, the output voltage $\mathrm{V}_{\text {ref }}$ is given by

$$
\begin{gathered}
V_{r e f}=V_{G S}+\frac{V_{X}}{R_{2}} V_{B E}+V_{o f} \\
V_{r e f}=n V_{T} \ln \left(\frac{n K_{4} K_{6}}{K_{5} K_{7}}\right)+\frac{V_{B E}}{R_{2} g m_{11}}+V_{o f}
\end{gathered}
$$

The derivative of $\mathrm{V}_{\text {ref }}$ over temperature is given by

$$
\frac{\delta V_{r e f}}{\delta T}=\eta \frac{k}{q} \ln \left(\frac{n K_{4} K_{6}}{K_{5} K_{7}}\right)+\frac{K_{N}}{R_{2} g m_{11}}
$$

where $\mathrm{K}_{\mathrm{N}}$ is the negative $\mathrm{TC}$ of $\mathrm{V}_{\mathrm{BE}}$. A zero $\mathrm{TC}$ can be obtained since the two terms of (12) can cancel each other with proper design, and hence $\mathrm{V}_{\text {ref }}$ would be stable across a temperature variation range. The absolute value of the negative $\mathrm{TC}$ is reduced by $1 / \mathrm{gm}_{11} \mathrm{R}_{2}$ times compared with the traditional current-mode bandgap voltage reference. So the PTAT compensation factor of $\left(\mathrm{K}_{4} \mathrm{~K}_{6} \mathrm{~K}_{5} \mathrm{~K}_{7}\right)$ can also be reduced, which helps reduce the power and area of the PTAT circuit.

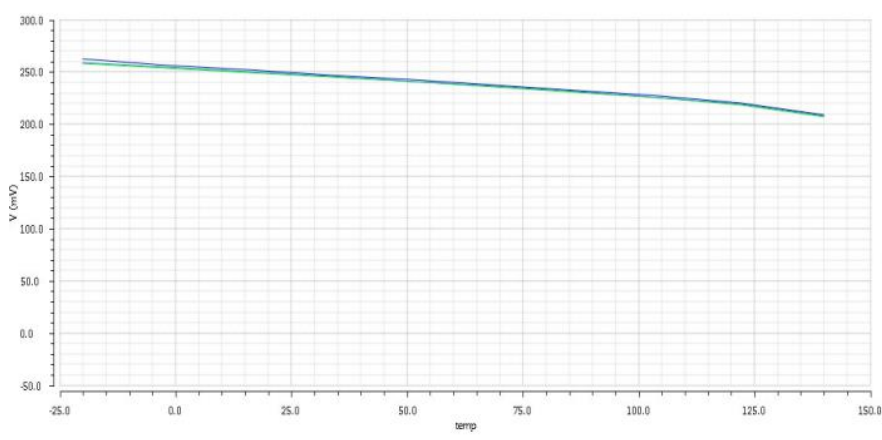

Fig. 4. Practically measured $\mathrm{V}_{\text {ref }}$ versus temperature

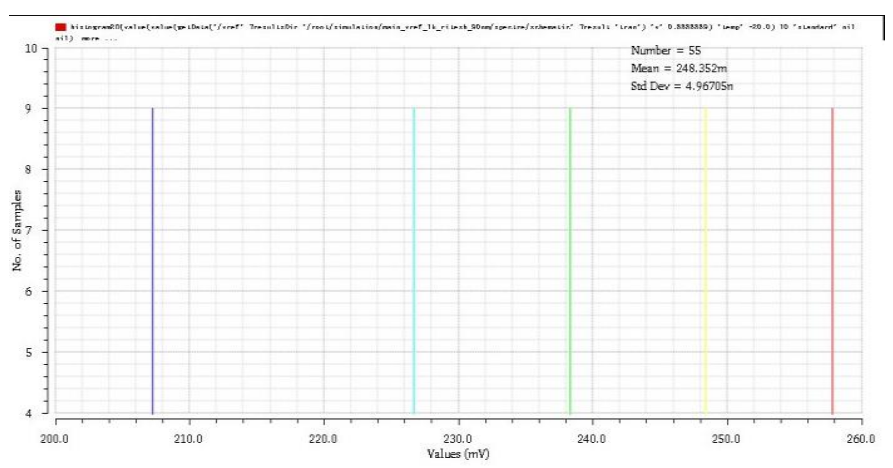

Fig. 5. Histogram simulation result of supply voltage.

\section{RESULT}

The proposed sub-BGR schematic is developed using cadence virtuoso tool in a standard 90-nm CMOS. Simulated output using Spectre simulator as shown in fifigures. Fig 4. shows the measured output voltage $\mathrm{V}_{\text {ref }}$ versus temperature variance range. Fig 5. shows the histogram simulation results of $\mathrm{V}_{\text {ref }}$ and TC. The average $\mathrm{V}_{\text {ref }}$ and standard deviation are $248.352 \mathrm{mv}$ and $4.967 \mathrm{nV}$ respectively, leading to a variation coefficient i.e minimum. Fig 6 and 7 show The spectral power of the sub-BGR proposed in Fig 6 shows Power versus voltage range from $0 \mathrm{v}$ to $2 \mathrm{v}$ and Power variation is nearly constant from $0.7 \mathrm{v}-2 \mathrm{v}$. Fig 7 . show the average power consumption and standard deviation are $457 \mathrm{pw}$ and $231 \mathrm{pw}$ respectively, with range of temperature $-20^{\circ} \mathrm{C}-140^{\circ} \mathrm{C}$.

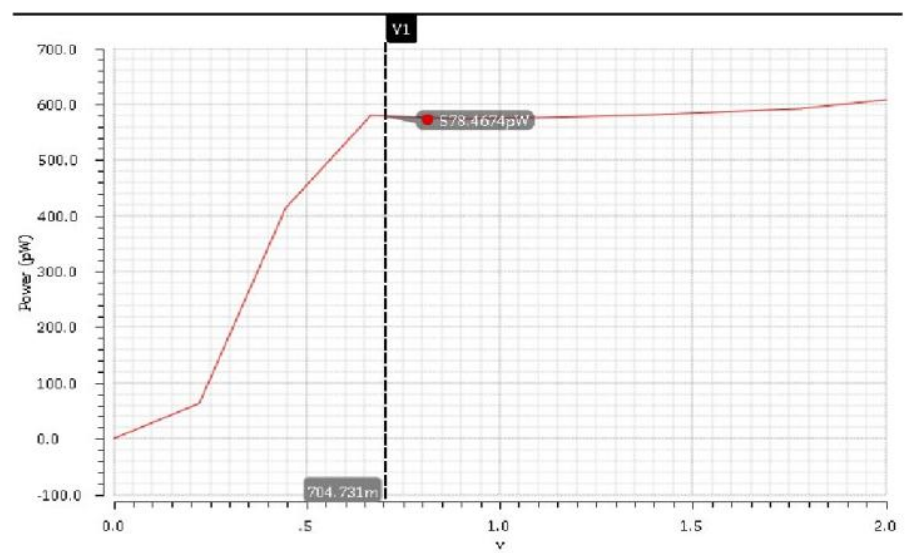

Fig. 6. Practically measured spectral power versus supply voltage.

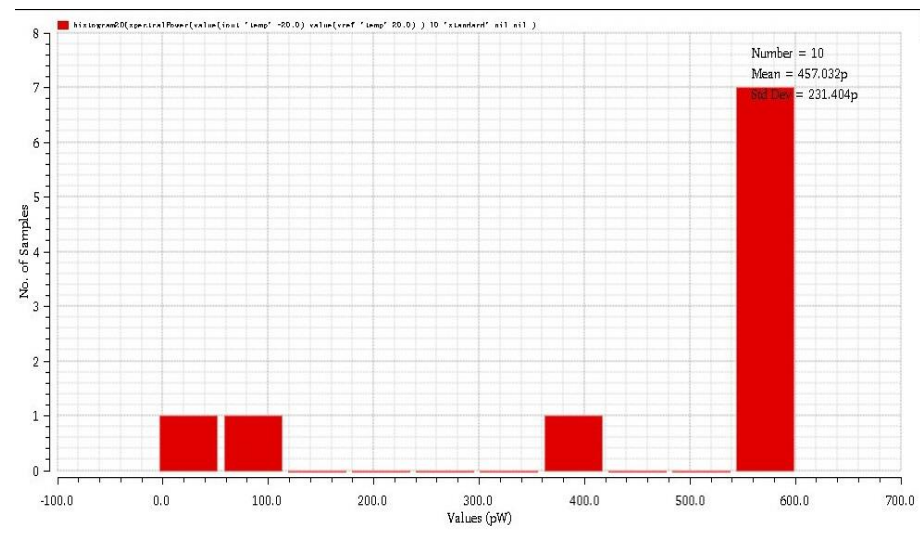

Fig. 7. Histogram simulation result of power.

\section{REFERENCES}

[1] D. Albano, F. Crupi, F. Cucchi, and G. Iannaccone, “A sub-T/q voltage reference operating at $150 \mathrm{mV}$ ", IEEE Trans. Very Large Scale Integr. (VLSI) Syst., vol. 23, no. 8, pp. 1547-1551, Aug. 2015.

[2] Jinghui An, Chenjian Wu, Dacheng Xu, “A Wide Temperature Range 4.6 ppm/C Piecewise Curvature-Compensated Bandgap Reference With No Amplififiers" ,International Conference on IC Design and Technology (ICICDT), Aug. 2019.

[3] H. Banba et al., "A CMOS bandgap reference circuit with sub-1-V operation," IEEE J. Solid-State Circuits, vol. 34, no. 5, pp. 670-674, May 1999.

[4] M. Kim and S. Cho, "A 0.8 V, $37 \mathrm{nW}, 42 \mathrm{ppm} / \mathrm{C}$ sub-bandgap voltage reference with PSRR of $81 \mathrm{~dB}$ and line sensitivity of $51 \mathrm{ppm} / \mathrm{V}$ in 
0.18 $\mu \mathrm{m}$ CMOS," in Proc. Symp. VLSI Circuits, Kyoto, Japan, pp. 144145, Jun. 2017.

[5] J. M. Lee et al., "A $29 \mathrm{nW}$ bandgap reference circuit," in IEEE Int. Solid State Circuits Conf. (ISSCC) Dig. Tech. Papers, pp. 1-3 ,Feb. 2015.

[6] A. Shrivastava, K. Craig, N. E. Roberts, D. D. Wentzloff, and B. H. Calhoun, "A $32 \mathrm{nW}$ bandgap reference voltage operational from $0.5 \mathrm{~V}$ supply for ultra-low power systems," in IEEE Int. Solid-State Circuits Conf. (ISSCC) Dig. Tech. Papers, pp. 13 , Feb. 2015.

[7] Y. Liu, C. Zhan, and L. Wang, "An ultralow power subthreshold CMOS voltage reference without requiring resistors or BJTs," IEEE Trans. Very Large Scale Integr. (VLSI) Syst., vol. 26, no. 1, pp. 201205, Jan. 2018

[8] L. Magnelli, F. Crupi, P. Corsonello, C. Pace, and G. Iannaccone, "A $2.6 \mathrm{nW}, 0.45 \mathrm{~V}$ temperature-compensated subthreshold CMOS voltage reference,” IEEE J. Solid-State Circuits, vol. 46, no. 2, pp. 465-474, Feb. 2011

[9] M. Seok, G. Kim, D. Blaauw, and D. Sylvester, "A portable 2transistor picowatt temperature-compensated voltage reference operating at $0.5 \mathrm{~V}$," IEEE J. Solid-State Circuits, vol. 47, no. 10, pp. 25342545, Oct. 2012.

[10] D. Wang, X. L. Tan, and P. K. Chan, "A 65-nm CMOS constant current source with reduced PVT variation," IEEE Trans. Very Large Scale Integr. (VLSI) Syst., vol. 25, no. 4, pp. 13731385, Apr. 2017.

[11] M.-E. Hwang and K. Roy, "A $135 \mathrm{mV} 0.13 \mathrm{~W}$ process tolerant 6T subthreshold DTMOS SRAM in $90 \mathrm{~nm}$ technology," in Proc. IEEE Custom Integr. Circuits Conf. (CICC), pp. 419422 Sep. 2008.

[12] B. Razavi, Design of Analog CMOS Integrated Circuits. New York, NY, USA: McGraw-Hill, 2001. 